TRANSACTIONS OF THE

AMERICAN MATHEMATICAL SOCIETY

Volume 182, August 1973

\title{
ON THE INTEGRALS OF PERRON TYPE
}

\author{
BY \\ P. S. BULLEN AND C. M. LEE
}

\begin{abstract}
An abstract derivate system is defined axiomatically, and then a naturally corresponding Perron integral theory is developed, unifying all the existing integral theories of Perron type of first order. A new scale of approximately mean-continuous integrals and a new scale of symmetric Cesàro-Perron integrals are obtained as examples of the general theory. Also, the $M Z$-integral and the $S C P$-integral are proved to be equivalent.
\end{abstract}

0 . Introduction. In calculus, usually two fundamental concepts of integration are introduced. One corresponds to the idea of Riemann integral as a limit of certain approximating sums and the other to that of Newton integral understood as an antiderivative. These two integrals are related by the fundamental theorem of integration calculus. However, they are not equivalent. Even the more powerful Lebesgue integral does not include the Newton integral. This leads to the problem of defining an integral which includes both Lebesgue and Newton integrals. Both Denjoy and Perron gave solutions to the problem by defining a $\mathscr{D}_{*}$-integral and a $P$-integral (see [22]), respectively. The approach to $\mathfrak{I}_{*}$-integral starts from the Lebesgue integral, and is a complicated (though beautiful) transfinite inductive process, successively using Cauchy extension and Harnack extension. On the other hand, the approach to $P$-integral does not use any Lebesgue theory and is a simple synthesis process of the fundamental concepts of integration in calculus.

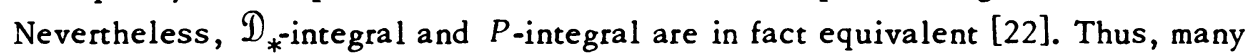
authors have adopted the simple Perron method in defining more general integrals; many of them have applications to the coefficient problem of trigonometric series. See, for example, Marcinkiewicz and Zygmund [15], James [10]-[12], Burkill [5], and Taylor [27].

We propose here to study various integrals of Perron type. We do this by obtaining a general Perron integral theory defined in an abstract "derivative system", which is assumed to satisfy only certain axioms $(\$ 1)$. Thus, we unify all

Received by the editors December 28, 1972.

AMS (MOS) subject classifications (1970). Primary 26A39; Secondary 26A24, 28A15.

Key words and phrases. Base, base mappings, lower derivate operator, derivative, legitimate mapping, inequality property, derivate system, major function, minor function, Perron integral, Cesàro-Perron integral, approximately mean-continuous integral, symmetric Cesàro-Perron integral, $M Z$-integral, $S C P$-integral. 
the integral theories of Perron type of first order known to us. In addition, the $P$ and $R$-integral theories of Romanovski [21] and the abstract integral the ory of Pfeffer [19] are contained in our general theory as particular cases $(\$ 4)$. We note that such kind of general abstract theory has been done for integrals of most of the other types. For example, Lebesgue integral has been defined in the abstract measure space, the integral of Riemann type in the divison space ([9] and [18]), the integral of Denjoy type in the Romanovski space ([21] and [26]).

The definition and the elementary properties of our integral are given in $\$ 2$, and convergence theorems similar to those of Lebesgue integral in $\$ 3$. A differential property and a characterization of integrability are given in $\$ 5$ for integrals defined in abstract derivate system in the real line. The latter is used to prove the equivalence of $M Z$-integral [15] and $S C P$-integral [5] in $\$ 6$, where a scale of symmetric $C P$-integrals and a scale of approximately mean-continuous integrals are also briefly stated as examples for our general theory.

1. Settings. Let $X$ be a given set, $\sigma$ a given collection of subsets of $X$. For $A \subset X$, define $\sigma_{A}$ by

$$
\sigma_{A}=\left\{A^{\prime} \mid A^{\prime} \in \sigma \text { and } A^{\prime} \subset A\right\}
$$

1.1. Base mappings. Let $A \in \sigma$. A subset $\beta$ of $\sigma_{A}$ will be called a base in $A$ if $A \in \beta$ and if for each $A^{\prime} \in \beta$ there exists a finite set of disjoint $A_{i} \in \beta$ with $A_{i} \cap A^{\prime}=\varnothing$ for each $i$ and $\left(\bigcup_{i} A_{i}\right) \cup A^{\prime}=A$. By a base mapping on $\sigma$ we mean a mapping $\mathscr{B}$ on $\sigma$ such that for each $A \in \sigma$, the image $\mathscr{B}(A)$ is a collection of bases in $A$ satisfying the following axioms:

( $\left.B_{1}\right) \beta_{1}, \beta_{2} \in \mathfrak{B}(A)$ implies $\beta_{1} \cap \beta_{2} \in \mathfrak{B}(A)$;

(B2) $\beta \in \mathcal{B}^{2}(A)$ and $A^{\prime} \in \beta$ imply that $\beta_{A^{\prime}}=\left\{A^{\prime \prime} \mid A^{\prime \prime} \in \beta\right.$ and $\left.A^{\prime \prime} \subset A^{\prime}\right\} \in \mathcal{B}\left(A^{\prime}\right)$;

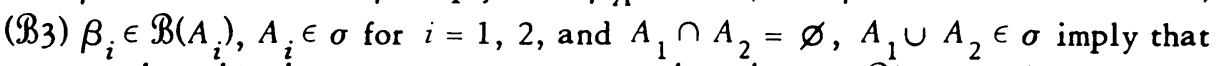
$\beta_{1} \oplus \beta_{2}=\left\{A_{1}^{\prime} \cup A_{2}^{\prime} \mid A_{i}^{\prime} \in \beta_{i}\right.$ for $i=1,2$, and $\left.A_{1}^{\prime} \cup A_{2}^{\prime} \in \sigma\right\} \in \mathfrak{B}^{\prime}\left(A_{1} \cup A_{2}\right)$.

1.2. Derivate operators. Let $A \in \sigma, \beta$ a subset of $\sigma_{A}$, and $\bar{O}$ a semivector space of (set) functions defined on $\beta$, where by a semivector space $\bar{C}$ we mean that $F_{1}, F_{2} \in \overline{\mathbb{O}}$ implies $a_{1} F_{1}+a_{2} F_{2} \in \overline{\mathbb{C}}$ for all real numbers $a_{1}, a_{2} \geq 0 . \mathrm{A}$ lower derivate operator on $\bar{C}$ is defined to be a mapping $\mathscr{D}$ with domain $\overline{\mathbb{C}} \times A$ such that, for each $v \in \overline{\mathbb{C}}$ and for each $x \in A$, the image $\underline{\mathfrak{I}}(v, x)=\underline{D} v(x)$ is an extended real value, and satisfying the following axioms:

$\left(\underline{I}_{1}\right)$ for all $x \in A, \underline{D}(0, x)=0$;

$\left(\underline{D}_{2}\right)$ for all $x \in A, v_{1}, v_{2} \in \overline{\mathbb{C}}, \underline{D}\left(v_{1}+v_{2}, x\right) \geq \underline{D}\left(v_{1}, x\right)+\underline{D}\left(v_{2}, x\right)$ whenever the addition on the right-hand side makes sense;

(D) 3$)$ for all $x \in A, v \in \overline{\mathbb{Z}}, a>0, \underline{D}(a v, x)=a \underline{D}(v, x)$; 
(D4) for all $x \in A, \underline{D}(-v, x) \leq-\underline{D}(v, x)$ whenever both $v$ and $-v$ are in $\bar{\complement}$. For each $v \in \overline{\mathcal{O}}, x \in A$, defining

$$
\overline{\mathfrak{D}}(-v, x)=-\underline{D}(v, x),
$$

we call $\overline{\mathfrak{D}}$ the upper derivate operator corresponding to $\underline{D}$. Letting $\underline{\mathbb{C}}=$ $\{v \mid-v \in \overline{\mathbb{O}}\}$, we see that $\underline{\mathbb{E}} \times A$ is the domain of $\overline{\mathfrak{D}}$. It is easy to see that $\overline{\mathfrak{D}}$ has properties $\left(\mathscr{D}_{1}\right)-(\mathfrak{D} 4)$, of which the meaning is immediate. Furthermore, for $v \epsilon$ $\mathrm{C}^{\circ} \cap \underline{\mathrm{O}}$

$$
\overline{\mathfrak{D}}(v, x) \geq \underline{\mathfrak{D}}(v, x), \quad \overline{\mathfrak{D}}(\alpha v, x)=\alpha \underline{\mathbb{D}}(v, x)
$$

for all $x \in A$ and $\alpha<0$.

If $\underline{D}(v, x)$ and $\bar{D}(v, x)$ are equal, we say that $v$ is $\mathscr{D}$-differentiable at $x$, and the common value, denoted by $\mathcal{D}(v, x)$ or $\mathscr{D} v(x)$ is called the $\mathfrak{D}$-derivative of $v$ at $x$; for example, clearly $\mathscr{D}(0, x)=0$ for all $x \in A$.

1.3. Legitimate mappings. Let $F$ be an extended real-valued (set) function defined on $\gamma$, a collection of subsets of $X . F$ is said to be superadditive on $\gamma$ if

$$
F\left(\bigcup_{i} A_{i}\right) \geq \sum_{i} F\left(A_{i}\right)
$$

for every finite collection $\left\{A_{i}\right\}$ of disjoint sets from $\gamma$ for which $\bigcup_{i} A_{i} \in \gamma$ and the additions $\Sigma_{i} F\left(A_{i}\right)$ make sense. $F$ is defined to be subadditive if and only of $-F$ is superadditive. If $F$ is both superadditive and subadditive, we say that $F$ is additive.

Given a base mapping $\mathscr{B}$ on $\sigma$, let $\bar{T}$ be a mapping such that for each $A \epsilon$ $\sigma, \beta \in \mathcal{B}(A)$, the image $\mathbb{T}(A, \beta)$ is a semivector space of real-valued functions defined on $\beta$. If $\mathbb{M}$ satisfies further the following axioms, we say that $\mathbb{M}$ is a legitimate mapping on $\sigma$ with base mapping $B$.

$\left(\bar{M}_{1}\right)$ For any $\beta_{1}, \beta_{2} \in \mathfrak{B}(A)$ with $\beta_{1} \subset \underline{\beta}_{2}, \bar{M}\left(A, \beta_{1}\right) \supset \bar{M}\left(A, \beta_{2}\right)$.

$\left(\bar{M}(2)\right.$ For any $\beta \in \mathcal{B}(A)$ and any $A^{\prime} \in \beta, \bar{M}(A, \beta) \mid A^{\prime}=\left\{M\left|\beta_{A^{\prime}}\right| M \in \bar{M}(A, \beta)\right\} \subset$ $\bar{M}\left(A^{\prime}, \underline{\beta}_{A^{\prime}}\right)$.

(M/3) For $A_{1}, A_{2}, \beta_{1}, \beta_{2}$, as in $\left(B_{3}\right)$, if $M_{i} \in \bar{M}\left(A_{i}, \beta_{i}\right)$ for $i=1,2$, then $M_{12} \in \bar{M}\left(A_{1} \cup A_{2}, \beta_{1} \oplus \beta_{2}\right)$, where $M_{12}\left(A^{\prime}\right)=M_{1}\left(A_{1}^{\prime}\right)+M_{2}\left(A_{2}^{\prime}\right)$ for any $A^{\prime}=$ $A_{1}^{\prime} \cup A_{2}^{\prime}$ in $\beta_{1} \oplus \beta_{2}$ with $A_{i}^{\prime} \in \beta_{i}, i=1,2$.

(M4) $M_{1}=M_{2}$ on $\beta$ and $M_{1} \in \bar{M}(A, \beta)$ imply that $M_{2} \in \bar{M}(A, \beta)$.

$(\bar{M} 5) \bar{M}(A, \beta)$ is closed under uniform sequence convergences in $\beta$ (i.e. if $F_{n} \in \bar{M}(A, \beta)$ for $n=1,2,3, \cdots$, and $F_{n} \rightarrow F$ uniformly in $\beta$, then $\left.F \in \bar{M}(A, \beta)\right)$.

1.4. Inequality properties. By an inequality property on set functions we mean a property $I$ satisfying the following axioms.

(I 1$)$ If $F_{1}$ and $F_{2}$ are two set functions defined on $\gamma$ and if both $F_{1}$ and $F_{2}$ satisfy the property $\bar{I}$ on $\gamma$, then $\alpha_{1} F_{1}+\alpha_{2} F_{2}$ satisfies $\bar{I}$ on $\gamma$ whenever 
$\alpha_{1} F_{1}+\alpha_{2} F_{2}$ makes sense, where $\alpha_{1}, \alpha_{2}$ are nonnegative real numbers.

$\left(\bar{I}_{2}\right)$ If a set function satisfies $\bar{I}$ on domains $\gamma_{1}, \gamma_{2}$, respectively, it does so on $\gamma_{1} \cap \gamma_{2}$ and $\gamma_{1} \cup \gamma_{2}$.

$\left(\bar{I}_{3}\right)$ If $F_{1}$ and $F_{2}$ are two set functions on $\gamma$ with $F_{1} \geq F_{2}$ and $F_{2}$ satisfies $\bar{I}$ on $\gamma$, then $F_{1}$ satisfies $\bar{I}$ on $\gamma$.

If $\bar{I}$ is an inequality property, we denote its dual property by $I$ and by this we mean that $F$ satisfies $\underline{I}$ if and only if $-F$ satisfies $\bar{I}$. We will come across two kinds in inequality properties in the examples considered later; one is defined by means of inequalities containing the lower derivates of functions; the other is defined by means of inequalities containing the function values.

1.5. Derivate systems. Let $\pi$ be a fixed collection of subsets of $X$ closed under countable set unions, i.e. $E_{n} \in \mathcal{N}$ for $n=1,2,3, \cdots$ imply that $\bigcup_{n} E_{n} \in \Re$. For convenience, we say that a property $p(x)$ is true almost everywhere (a.e.) in $A(\subset X)$ if $p(x)$ is true for all $x$ in $A$ except at most for points of a set in $\pi$.

Given a legitimate mapping $\bar{\pi}$ on $\sigma$ with a base mapping $\mathcal{B}$ and an inequality property $\bar{I}$, suppose that, for each $A \in \sigma, \beta \in \Re(A)$, there exists a lower derivate operator $\underline{D}_{A \beta}$ on $\bar{M}(A, \beta)$. If the following axioms are satisfied, we say that $(\mathbb{N}, \underline{D}, \mathfrak{B}, \bar{\Re}, \bar{I})$ is a derivate system on $\sigma$.

$\left(\underline{D} \bar{M}_{1}\right)$ For $A_{1} \in \sigma, A_{2} \in \sigma_{A_{1}}, M_{i} \in \bar{M}\left(A_{i}, \beta_{i}\right), i=1,2, M_{1}=M_{2}$ on $\beta_{1} \cap \beta_{2}$, one has $\underline{D}_{A_{1} \beta_{1}}\left(M_{1}, x\right)=\underline{D}_{A_{2} \beta_{2}}\left(M_{2}, x\right)$ for each $x \in A_{2}$.

$\left(\bar{D}_{\bar{M}}\right)$ If $M \in \bar{M}(A, \beta)$ with $\underline{D}_{A \beta}(M, x) \geq 0$ a.e. in $A$ and $M$ satisfies the inequality property $\bar{I}$, then $M \geq 0$ on $\beta$.

Note that by axiom $\left(\mathfrak{D}_{\bar{M}}\right.$ ), we can always (without any ambiguity) write $\underline{D}(M, x)$ instead of $\underline{D}_{A \beta}(\bar{M}, x)$.

2. The integral. Let $X$ be a given set and $\sigma$ a given collection of subsets of $X$, and let $P=(\pi, \underline{D}, \Re, \pi, \bar{I})$ be a derivate system on $\sigma$. If we need other derivate systems on $\sigma$, we will denote them by $P_{1}=\left(\bar{\pi}^{1}, \mathscr{D}_{1}, \Re_{1}, \pi_{1}, \bar{I}_{1}\right)$ etc.

2.1. Major and minor functions. Let $A \in \sigma, \beta \in \mathfrak{B}(A)$ and $f$ be an extended real-valued function defined and finite a.e. in $A$. A function $M$ is a $P$-major function of $f$ on $A$ with base $\beta$, written $M \in \mathbb{M}_{f}(A, \beta)$, if

$\left(\bar{\pi}_{1}\right) M \in \bar{\Pi}(A, \beta)$;

$\left(\overline{\mathscr{N}}_{2}\right) \underline{D}(M, x) \geq f(x)$ a.e. in $A$;

$\left(\bar{M}_{3}\right) \bar{M}$ satisfies $\bar{I}$.

A function $m$ is a $P$-minor function of $f$ on $A$ with base $\beta$, written $m \epsilon$ $\underline{M}_{f}(A, \beta)$, if $-m \in \bar{M}_{-f}(A, \beta)$. We will write

$$
\underline{\mathbb{R}}(A, \beta)=\{-M \mid M \in \overline{\mathbb{M}}(A, \beta)\} \text {. }
$$


It is easy to see that $m \in \underline{M}_{f}(A, \beta)$ if and only if

( $\underline{1} 1) m \in \underline{\mathbb{N}}(A, \beta)$;

( $\underline{2} 2) \overline{\mathscr{T}}(m, x) \leq f(x)$ a.e. in $A$;

(m) $m$ satisfies $I$.

The following lemma is fundamental for our theory. We omit the proof, which is trivial by $\left(T / T^{2}\right)$.

Lemma 1. For $M \in \bar{M}_{f}(A, \beta), m \in \underline{M}_{f}(A, \beta), M-m$ is superadditive and nonnegative on $\beta$. In particular, $M(A) \geq m(A)$.

2.2. The definition of the integral. If both $\overline{\mathbb{R}}_{f}(A, \beta)$ and $\underline{\mathbb{M}}_{f}(A, \beta)$ are not empty and

$$
\inf \left\{M(A) \mid M \in \overline{\mathbb{M}}_{f}(A, \beta)\right\}=\sup \left\{m(A) \mid m \in \underline{\mathbb{M}}_{f}(A, \beta)\right\} \neq \pm \infty,
$$

then we say that $f$ is $P$-integrable on $A$ with base $\beta$, and the common value, '-. noted by $(P)-\int_{A}^{\beta} f$, is called the P-integral of $f$ on $A$ with base $\beta$. The set of $P$-integrable functions on $A$ with base $\beta$ will be denoted by $P(A, \beta)$.

The following lemma is an immediate consequence of Lemma 1.

Lemma 2. $f \in P(A, \beta)$ if and only if for each $\epsilon>0$ there exist $M \in \bar{\pi}_{f}(A, \beta)$ and $m \in \prod_{f}(A, \beta)$ with $M(A)-m(A)<\epsilon$.

Lemma 3. Let $\beta_{1}, \beta_{2} \in \mathfrak{B}(A)$ with $\beta_{1} \subset \beta_{2}$. If $f \in P\left(A, \beta_{2}\right)$, then $f \in P\left(A, \beta_{1}\right)$ and two integrals are equal. In particular if $\beta, \beta^{\prime} \in \mathcal{B}(A)$ and $f \in P(A, \beta)$ and $f \epsilon$ $P\left(A, \beta^{\prime}\right)$, then $(P)-\int_{A}^{\beta} f=(P)-\int_{A}^{\beta^{\prime}} f$.

This lemma follows from $\left(\bar{\pi}_{1}\right),\left(T \bar{M}_{1}\right)$ and $\left(B_{1}\right)$ immediately. Henceforward, we can often without any ambiguity leave the base unspecified. We also note that if $f=g$ a.e. in $A$ and $f \in P(A, \beta)$, then $g \in P(A, \beta)$, and the integral of $f$ is equal to the integral of $g$.

2.3. Elementary properties of the integral. The following properties are easily proved.

(A) $P(A, \beta)$ is a vector space and the $P$-integral is linear on $P(A, \beta)$.

(B) If $f \in P(A, \beta)$ and $A^{\prime} \in \beta$, then $f \in P\left(A^{\prime}, \beta_{A}^{\prime}\right)$. Furthermore if $A_{1}, A_{2} \epsilon$ $\beta$ with $A_{1} \cap A_{2}=\varnothing$ and $A_{1} \cup A_{2}=A$, then $\int_{A} f=\int_{A_{1}} f+\int_{A_{2}} f$.

(C) If $f \in P\left(A_{i}, \beta_{i}\right)$ for $i=1,2$, where $A_{1} \cap A_{2}=\varnothing$ and $A_{1} \cup A_{2} \in \sigma$, then $f \in P\left(A_{1} \cup A_{2}, \beta_{1} \oplus \beta_{2}\right)$ and $\int_{A_{1} \cup A_{2}} f=\int_{A_{1}} f+\int_{A_{2}} f$.

(D) Let $F \in \mathbb{M}(A, \beta) \cap \underline{M}(A, \beta)$ and satisfy both $\bar{I}$ and $\underline{I}$. If $\mathscr{T} F(x)$ exists and is finite a.e. in $A$, then $\mathscr{D} F \in P(A, \beta)$ and $\int_{A} \mathscr{D} F=F(A)$. 
If $f \in P(A, \beta)$, then by (B), we see that $f \in P\left(A^{\prime}, \beta_{A^{\prime}}\right)$ for each $A^{\prime} \in \beta$. Define $F\left(A^{\prime}\right)=\int_{A^{\prime}} f$ for each $A^{\prime} \in \beta$. F is called the primitive of $f$ on $A$ with base $\beta$. By (B), we know that $F$ is additive on $\beta$, so that one gets easily the following properties.

(E) Let $f \in P(A, \beta)$ with primitive $F$, and $M \in \bar{M}_{f}(A, \beta), m \in \mathbb{M}_{f}(A, \beta)$. Then $M-F, F-m$ are both superadditive and nonnegative on $\beta$.

(F) If $F$ is a primitive of $f \in P(A, \beta)$, then $F \in \bar{M}(A, \beta) \cap \underline{M}(A, \beta)$.

(G) Let $P_{i}=\left(\mathbb{N}^{i}, \mathscr{D}_{i}, \Re_{i}, \Re_{i}, I_{i}\right)$ be a derivate system on $\sigma$ for $i=1$, 2. Suppose that $\bar{M}^{1}(A, \beta) \subset \bar{\pi}^{2}(A, \beta), \pi_{1} \subset \Re_{2}$, and that each function satisfying $\bar{I}_{1}$ satisfies $\bar{I}_{2}$, and $\underline{D}_{1}(M, x) \leq \underline{\mathbb{D}}_{2}(M, x)$ for each $M \in \bar{M}^{1}(A, \beta)$, then

$$
P_{1}(A, \beta) \subset P_{2}(A, \beta) \text { and }\left(P_{1}\right)-\int f=\left(P_{2}\right)-\int f
$$

for each $f \in P_{1}(A, \beta)$.

We remark that $(F)$ follows from $\left(\bar{T}_{5}\right)$ and the following lemma, while the other properties follow directly from the definitions and previous lemmas.

Lemma 4. If $f \in P(A, \beta)$ with primitive $F$, then there exists a sequence $\left\{M_{k}\right\} \subset$ $\bar{M}_{f}(A, \beta)$ and a sequence $\left\{m_{k}\right\} \subset \mathbb{M}_{f}(A, \beta)$ such that $0 \leq M_{k}\left(A^{\prime}\right)-F\left(A^{\prime}\right)<1 / k$ and $0 \leq F\left(A^{\prime}\right)-m_{k}\left(A^{\prime}\right)<1 / k$ for each $A^{\prime} \in \beta$.

3. Convergence theorems. With some reasonable restrictions on the derivate system, we now obtain some convergence theorems for our integral similar to those for the Lebesgue integral. Our proofs (cf. [19]) are different from the usual ones for various known integrals of Perron type since we do not appeal to the corresponding theorems for Lebesgue integral. In fact, our general abstract setting does not even contain any concept related to the Lebesgue integral. Throughout this section, we assume that the derivate system $P=(\bar{\eta}, \underline{D}, \mathfrak{B}, \eta, \bar{I})$ satisfies the following additional axioms.

$\left(\bar{M} 5^{\prime}\right)$ If $\left\{M_{n}\right\} \subset \bar{M}(A, \beta)$ and $M_{n} \uparrow M$ on $\beta$, then $M \in \bar{M}(A, \beta)$, where by $M_{n} \uparrow M$ on $\beta$ we mean that $M_{n}\left(A^{\prime}\right) \leq M_{n+1}\left(A^{\prime}\right)$ for each $A^{\prime} \in \beta$ and for $n=1,2,3, \cdots$, and $M_{n}\left(A^{\prime}\right) \rightarrow M\left(A^{\prime}\right)$ as $n \rightarrow+\infty$ for each $A^{\prime} \in \beta$.

$\left(\underline{D} \overline{\mathbb{M}}_{3}\right)$ For $M \in \overline{\mathbb{M}}(A, \beta)$ with $M \geq 0$ on $\beta, \underline{\mathcal{D}}(M, x) \geq 0$ for all $x \in A$.

Note that $\left(\mathcal{D}_{\bar{\pi}} \bar{\pi}_{3}\right)$ is a very natural axiom, while if it should be true that each function in $\bar{\pi}(A, \beta)$ is additive on $\beta$ then $(\bar{\pi} 5)$ implies $\left(\bar{\pi} 5^{\prime}\right)$.

Theorem 1. Suppose that $f_{n}, f$ are functions defined and finite almost everywhere in $A$ and $f_{n} \in P(A, \beta)$ for $n=1,2,3, \cdots$, and $f_{n}(x) \uparrow f(x)$ almost everywhere in $A$. Then $f \in P(A, \beta)$ and $\lim _{n} \int f_{n}=\int f$.

Proof. First, note that if $g(x) \leq b(x)$ almost everywhere in $A$ and $g, b \in$ 
$P(A, \beta)$, then $\int g \leq \int b$. This, in fact, follows directly from the definition of the integral.

Now, let $F_{n}$ be the primitive of $f_{n}$ for $n=1,2,3, \ldots$. Then by property (F), $F_{n} \in \bar{M}(A, \beta) \cap \underline{M}(A, \beta)$. For each $\epsilon>0$, choose $M_{n} \in \bar{M}_{f_{n}}(A, \beta)$ such that $0 \leq M_{n}-F_{n}<\epsilon / 2^{n}$ for $n=1,2,3, \cdots$, which is possible by Lemma 4 . In $\beta$, let $N_{1}=M_{1}$, and for $n \geq 2, N_{n}=M_{n}+\sum_{i=1}^{n-1}\left(M_{i}-F_{i}\right)$. Then $N_{n} \in \bar{M}(A, \beta)$ and $N_{n} \geq$ $M_{n}$. Furthermore,

$$
\begin{aligned}
N_{n+1} & =M_{n+1}+N_{n}-F_{n} \\
& \geq M_{n+1}+N_{n}-F_{n+1} \text { as } F_{n} \leq F_{n+1} \\
& \geq N_{n} \text { as } M_{n+1} \geq F_{n+1} .
\end{aligned}
$$

Thus, $N_{n} \uparrow N$ in $\beta$. By $\left(\bar{\pi}^{\prime}\right), N \in \bar{M}(A, \beta)$. By $(\bar{I} 3), N$ satisfies $\bar{I}$ since $M_{n}$ does and $N \geq N_{n} \geq M_{n}$. Moreover, by $(\underline{D} 2)$ and (D) 3 ), one easily shows that $\underline{D}(N, x) \geq f(x)$ almost everywhere in $A$. Thus, we have proved that $N \in \mathbb{M}_{f}(A, \beta)$.

Furthermore, since

$$
\begin{aligned}
N_{n}(A) & \leq M_{n}(A)+\sum_{i=1}^{n-1} \epsilon / 2^{i} \\
& \leq F_{n}(A)+\epsilon / 2^{n}+\sum_{i=1}^{n-1} \epsilon / 2^{i}=F_{n}(A)+\sum_{i=1}^{n} \epsilon / 2^{i} \text { for } n=2,3,4, \ldots,
\end{aligned}
$$

one has

$$
\inf \left\{M(A) \mid M \in \bar{\pi}_{f}(A, \beta)\right\} \leq N(A) \leq \lim _{n} F_{n}(A)+\epsilon .
$$

As $\epsilon$ is arbitrary, we see that

$$
\inf \left\{M(A) \mid M \in \bar{M}_{f}(A, \beta)\right\} \leq \lim _{n} F_{n}(A) .
$$

Similarly, using minor functions, one can prove that $\underline{\pi}_{f}(A, \beta)$ is not empty and

$$
\sup \left\{m(A) \mid m \in \mathbb{M}_{f}(A, \beta)\right\} \geq \lim _{n} F_{n}(A) .
$$

Thus, by Lemma $1, f \in P(A, \beta)$ and

completing the proof.

$$
\int f=\lim _{n} F_{n}=\lim _{n} \int f_{n}
$$

Theorem 2. Suppose that $f_{n}, f$ are functions defined and finite almost everywhere in $A$, and $f_{n} \in P(A, \beta)$ for $n=1,2,3, \ldots$. Further, suppose that lim inf ${ }_{n} f_{n}(x)=f(x)$ almost everywhere in $A$. If

then

$$
\inf \left\{M(A) \mid M \in \bar{\pi}_{\inf _{n} f_{n}}(A, \beta)\right\}>-\infty
$$

$$
\inf \{M(A) \mid M \in \bar{\pi} f(A, \beta)\} \leq \lim \inf _{n} \int_{A} f_{n} .
$$

Proof. Let $g_{n}(x)=\inf _{k \geq n} f_{k}(x)$ almost everywhere in $A$. 
Then $f_{k} \geq g_{n}$ for each $k \geq n$ and $g_{n}(x) \uparrow f(x)$ a.e. in $A$. Hence

$$
\inf \left\{M(A) \mid M \in \overline{\mathbb{M}}_{\mathbb{B}_{n}}(A, \beta)\right\} \leq \inf \left\{M(A) \mid M \in \bar{\pi}_{f_{k}}(A, \beta)\right\}
$$

for $k \geq n$ since $\bar{M}_{f_{k}}(A, \beta) \subset \bar{M}_{g_{n}}(A, \beta)$ by $f_{k} \geq g_{n}$. Thus,

for $n=1,2,3, \ldots$ Hence

$$
\inf \left\{M(A) \mid M \in \bar{M}_{g_{n}}(A, \beta)\right\} \leq \inf _{k \geq n} \int_{A} f_{k}
$$

$$
\lim _{n}\left[\inf \left\{M(A) \mid M \in \bar{M}_{g_{n}}(A, \beta)\right\}\right] \leq \lim \inf _{n} \int_{A} f_{n} .
$$

Now, as $g_{n} \uparrow f$ and $\inf \left\{M(A) \mid M \in \bar{M}_{g 1}(A, \beta)\right\}>-\infty$, following an argument similar to that in the proof of Theorem 1 , one proves easily that

and the proof is hence completed.

$$
\lim _{n}\left[\inf \left\{M(A) \mid M \in \bar{M}_{B_{n}}(A, \dot{\beta})\right\}\right] \geq \inf \left\{M(A) \mid M \in \bar{M}_{f}(A, \beta)\right\}
$$

Theorem 3. Suppose that $h, g, f_{n} \in P(A, \beta)$ and $g(x) \leq f_{n}(x) \leq b(x)$ almost everywhere in $A$ for $n=1,2,3, \ldots$. If $f$ is a function defined and finite almost everywbere in $A$ with $\lim _{n} f_{n}(x)=f(x)$ almost everywbere in $A$, then $f \in P(A, \beta)$ and $\int f=\lim _{n} \int f_{n}$.

Proof. Let $\phi_{n}=f_{n}-g, \phi=f-g, \psi=b-g$. Then $0 \leq \phi_{n} \leq \psi$ almost everywhere in $A$, so that $0 \leq \inf _{n} \phi_{n} \leq \sup \phi_{n} \leq \psi$ almost everywhere in $A$. Hence

and

$$
\inf \left\{M(A) \mid M \in \bar{M}_{\inf _{n} \phi}(A, \beta)\right\}>-\infty
$$

$$
\sup \left\{m(A) \mid m \in \underline{\mathbb{M}}_{\sup _{n} \phi_{n}}(A, \beta)\right\}<+\infty .
$$

By Theorem 2 and its dual, we see that

$$
\begin{aligned}
& a \equiv \inf \left\{M(A) \mid M \in \overline{\mathbb{M}}_{\phi}(A, \beta)\right\} \leq \underset{n}{\lim \inf } \int_{A} \phi_{n}, \\
& b \equiv \sup \left\{m(A) \mid m \in \underline{\mathbb{M}}_{\phi}(A, \beta)\right\} \geq \underset{n}{\lim \sup } \int_{A} \phi_{n}
\end{aligned}
$$

as $\phi_{n}(x) \rightarrow \phi(x)$ almost everywhere in $A$. By Lemma 1 , we have $a \geq b$, and in general we have $\lim$ sup $\geq \lim$ inf, so that $\phi \in P(A, \beta)$ and $\int \phi=\lim _{n} \int \phi_{n}$.

Now, $f=\phi+g$, so that by property $(A)$, we see that $f \in P(A, \beta)$ and $\int f=$ $\lim _{n} \int f_{n}$, completing the proof.

4. Some general integrals as particular cases. In [21], Romanovski has defined a $P$-integral and a $R$-integral in certain abstract spaces, and in [19], Pfeffer has defined another integral in topological spaces with certain local net convergence structure. We show briefly how all these integrals can be obtained from our theory.

Let $(X, \sigma, \mu)$ be a Romanovski space. For this and for some of the following terminologies and notations, we refer to [21] and [26]. For each $A \in \sigma$, let $\mathfrak{B}(A)=$ 
$\left\{\sigma_{A}\right\}$ and $\bar{\pi}\left(A, \sigma_{A}\right)=\bar{\pi}(A)=\left\{F \mid F\right.$ is additive on $\sigma_{A}$ and is continuous from interior on $A\}$.

Then $\mathcal{B}$ is a base mapping and $\bar{M}$ is a legitimate mapping on $\sigma$ according to the definition in $\S 1$.

For a function $F$ defined on $\sigma_{A}$, let

$$
\underline{D} F(x)=\lim _{A^{\prime} \in \sigma_{A}, x \in \bar{A}^{\prime}} \frac{F\left(A^{\prime}\right)}{\mu\left(A^{\prime}\right)},
$$

where $\bar{B}$ denotes the closure of $B$ in $X$. We say that $F$ satisfies $\bar{I}$ on $\sigma_{A}$ if $\underline{D} F(x)>-\infty$ except perhaps for points $x$ of countably many boundaries of sets in $\sigma$. Then $\bar{I}$ and $\underline{A C G}$ (defined in [21]) are both inequality properties as defined in $\oint_{1}$.

Let $\pi=$ the family of all subsets of $X$ with zero $\mu$-measure, and let $P=$ $(\bar{\pi}, \underline{D}, \Re, \pi, \bar{I}), R=(\bar{\pi}, \underline{D}, \Re, \pi, \underline{A C G}$. It follows from lemmas on pp. 92 and 95 in [21] that both $P$ and $R$ are derivate systems on $\sigma$. The $P$-integral and $R$-integral are just those defined by Romanovski in [21].

By the theorem on p. 77 in [21], we see easily that both $P$-and $R$-integrals have a differential property similar to that given in Theorem 4, next section. Whether there is a result similar to Theorem 5 below for the $P$-integral here is an open question. The proof of Theorem 5 depends on the Zahorski function on the real line. Thus, if such a function could be constructed on an arbitrary Romanovski space, the question could be settled.

Now, turning to the Pfeffer integral, we denote the $P$ in [19] by $X$, and let $(X, \sigma, \kappa, G)$ be the setting there. Noticing the meanings of the operators \# and * in [19], we define the derivate system $P=(\bar{\pi}, *, \mathcal{B}, \Re, \bar{l})$ on $\sigma$ as follows. For each $A \in \sigma$, define $\mathfrak{B}(A)=\left\{\sigma_{A}\right\}$ and $\bar{M}\left(A, \sigma_{A}\right)=\bar{M}(A)=\{F \mid F$ is a function defined and superadditive on $\sigma_{A}$, and there exists a countable set $Z_{F} \subset X$ such that ${ }_{\sharp} F(x, A) \geq 0$ for each $x \in Z_{F} \cup\{\infty\}$ and ${ }_{\#}(-G)(x, A) \geq 0$ for each $\left.x \in Z_{F}\right\}$. $\pi$ is defined to be the family of all countable subsets of $X$. By $F$ to satisfy the property $\bar{I}$ on $\sigma_{A}$, we mean that $* F(x, A)>-\infty$ for all $x \in \bar{A} \sim Z_{F}$. That $P$ such defined is in fact a derivate system on $\sigma$ follows from Lemma 5.9 in [19]. The P-integral is just that defined by Pfeffer in [19]. Whether this $P$-integral has a differential property requires further investigation.

5. Further properties of the integral on the real line. Throughout this section, let $X$ be the real line, and $\sigma$ the family of all bounded half-open intervals like $[a, b[=\{x \mid a \leq x<b\}$. We are going to obtain a differential property of the integral and a characterization of integrability and also a very general integration by parts formula for the integral corresponding to an abstract derivate system on $\sigma$.

Let $P=(\pi, \underline{D}, \Re, \pi, \bar{I})$ be a derivate system on $\sigma$. Furthermore, we assume that for each $\beta \in \mathfrak{B}\left(\left[a, b[)\right.\right.$, the set $B=\bigcup_{A \in \beta} \dot{A}$ is of measure $b-a$, where $\dot{A}$ 
denotes the endpoints of the interval $A$. Also, we assume that each $M \epsilon$ $\bar{\pi}([a, b[, \beta)$ is additive on $\beta$.

Then the interval function $M$ on $\beta$ is in one-to-one correspondence to the point function $M^{*}$ on $B$ as follows:

$$
\begin{aligned}
M^{*}(x) & =M([a, x[) & & \text { for each } x \in B \sim\{a\}, \\
& =0 & & \text { for } x=a, \\
M([x, y[) & =M^{*}(y)-M^{*}(x) & & \text { for each }[x, y[\in \beta .
\end{aligned}
$$

Should no ambiguities arise, we will not distinguish the interval functions $M$ on $\beta$ and the point functions $M^{*}$ on $B$. Sometimes, we also call $B$ a base in $[a, b]$. Note that by the remark at the beginning of $\S_{3}, P$ also satisfies the axiom $\left(\bar{\pi} 5^{\prime}\right)$. We assume furthermore that $P$ satisfies the following additional axioms.

(D) ) Each $\mathscr{D}_{M}$ is Lebesgue measurable.

(D6) $\underline{D}(M, x) \geq \underline{D}(M, x)$, where $\underline{D}(M, x)$ denotes the ordinary lower derivate of $M$ at $x$, i.e.

$$
\underline{D}(M, x)=\underline{D} M(x)=\lim _{x \in[a, b],[a, b[\epsilon \sigma} \inf _{A} \frac{M([a, b[)}{b-a}
$$

if $M$ is a function defined and additive on $\sigma_{A}$.

Theorem 4. Suppose that $f \in P(A, \beta)$ with primitive $F$. Then $\mathscr{D} F(x)$ exists and is finite almost everywhere in $A$.

Proof Let $k, \epsilon$ be arbitrary given positive numbers. By Lemma 2 there exists $M \in \bar{M},(A, \beta), m \in \underline{\mathbb{M}}_{f}(A, \beta)$ such that $M(A)-m(A)<k \epsilon$, and also $M(A)-F(A)<$ $k \epsilon$. Let $E_{0}$ be the set of points $x$ for which at least one of the following inequalities $\underline{D}_{M}(x) \geq f(x), \overline{\mathfrak{D}}_{m}(x) \leq f(x)$ fails to hold. Then $E_{0}$ is of measure zero. Observe that by property $(F), F \in \bar{M}(A, \beta) \cap \underline{\mathbb{M}}(A, \beta)$ so that $M-F \in \bar{M}(A, \beta)$. Hence by $(\mathbb{D} 5), \underline{D}(M-F)$ is measurable, so that the set $E_{k}$ of points $x$ in $A$ on which $\underline{D}(M-F, x) \geq k$ is measurable. We prove that $\mu\left(E_{k}\right)<\epsilon$ as follows, where $\mu$ is the Lebesgue measure on the real line.

Let $B=\bigcup_{A \in \beta} \dot{A}$. Consider $M, F$ as point functions on $B$, and define $R_{1}(x)=M(x)-F(x)$ for $x \in B$, and $R_{1}(x)=\sup _{y} \in B \cap[a, x] R_{1}(y)$ for $x \in A \sim B$, where $a$ is the left endpoint of $A$. Then $R_{1}$ is monotone increasing in $A$, and further $R_{1}$ is in $\bar{M}(A, \beta)$ by $(\bar{M} 4)$. Thus, $D\left(R_{1}, x\right)$, and hence $\mathscr{D}\left(R_{1}, x\right)$ by $(\mathscr{D} 6)$, exists and is finite almost everywhere in $A$, and

$$
(L)-\int_{A} \mathcal{D}\left(R_{1}, x\right) d x=(L)-\int_{A} D\left(R_{1}, x\right) d x \leq R_{1}(A)=M(A)-F(A)<k \epsilon,
$$

where $(L)$ denotes that the integral concerned is the Lebesgue integral. But

$$
(L)-\int_{A} \underline{D}\left(R_{1}, x\right) d x \geq(L)-\int_{E_{k}} \underline{\mathscr{D}}\left(R_{1}, x\right) d x \geq k \mu\left(E_{k}\right),
$$


So that $\epsilon>\mu\left(E_{k}\right)$, which is what we want to prove.

Now, for $x \notin E_{k} \cup E_{0}$,

$$
\underline{D}(F, x) \geq \underline{D}(M, x)-\overline{\mathscr{D}}(R, x) \geq f(x)-k .
$$

As $k$ and $\epsilon$ are arbitrary, it follows that $\mathscr{I}(F, x) \geq f(x)$ almost everywhere in $A$.

In a like manner, using minor functions, we can prove that $\overline{\mathfrak{T}} F(x) \leq f(x)$ almost everywhere in $A$. Then it follows that $\mathscr{T} F(x)$ exists and $\mathscr{T} F(x)=f(x)$ almost everyin $A$, completing the proof.

Corollary 1. Let $f \in P(A, \beta)$. Then $f$ is measurable in $A$.

Corollary 2. Let $f \in P(A, \beta), M \in \overline{\mathbb{M}}_{f}(A, \beta), m \in \underline{M}_{f}(A, \beta)$. Then $\mathfrak{D}_{M}(x)$ and $\mathfrak{D}_{m}(x)$ exist and are finite almost everywhere in $A$.

Suppose that the above derivate system $P$ satisfies in addition the following two axioms. We are going to obtain a characterization of integrability following the line of McGregor in [16].

(N) Each function $M$ continuous in $[a, b]$ belongs to $\bar{M}([a, b[, \beta)$ in the sense that the function $M^{*}\left(\left[x, y[)=M(y)-M(x)\right.\right.$ for $\left[x, y\left[\in \sigma_{[a, b}[\right.\right.$ belongs to $\pi([a, b[, \beta)$.

(I4) Let $\mathcal{C} \subset \mathcal{r}$ be closed under finite set unions. A function $F$ satisfies the inequality property $\bar{I}$ if and only if $\underline{\mathscr{I}} F(x)>-\infty$ except perhaps for points of a set in $\mathcal{C}$.

For convenience, we say that a property $p(x)$ is true nearly everywhere (n.e.) in $A$ if $p(x)$ is true for all $x$ in $A$ except at most for points of a set in $\mathcal{C}$. Note that the property $\bar{I}$ defined in $\left(I_{4}\right)$ is an inequality property, but not every inequality property is defined in this way.

Theorem 5. Let $f$ be a function finite almost everywbere in $A=[a, b[$. Then $f \in P(A, \beta)$ if and only if for each $\epsilon>0$, there exist functions $T, t$ such that

(i) $T \in \overline{\mathbb{M}}(A, \beta), t \in \underline{M}(A, \beta)$;

(ii) $\mathscr{D} T(x), \mathscr{D}_{t}(x)$ exist n.e. in $A$ and are finite a.e. in $A$;

(iii) $+\infty \neq \mathfrak{D}_{t}(x) \leq f(x) \leq \mathfrak{D}_{T}(x) \neq-\infty$ n.e. in $A$;

(iv) $T(A)-t(A)<\epsilon$.

Proof. It is clear that the conditions are sufficient. To see that the conditions are necessary, let $f \in P(A, \beta)$. Then for each $\epsilon>0$, take $M \epsilon \bar{\pi}_{f}(A, \beta), m \epsilon$ $\underline{M}_{f}(A, \beta)$ with $M(A)-m(A)<\epsilon / 2$, which is possible by Lemma 2 . By Corollary 2 to Theorem 4, $\mathfrak{D}_{M}(x)$, and $\mathfrak{D}_{m}(x)$ exist and are finite a.e. in $A$. Let $E$ be the subset of $A$ where at least one of $M, m$ fails to have a finite $D$-derivative. The set $E$ is of measure zero, so that there is a set $E_{1}$ of measure zero and of type $G_{\delta}$ such that $E \subset E_{1} \subset A$. 
Let $\omega$ be a point function defined on $[a, b]$ with the following properties:

(1) $\omega$ is $\mathrm{AC}$ on $[a, b]$;

(2) $\omega$ is differentiable in the ordinary sense;

(3) $\omega^{\prime}(x)=+\infty$ for $x \in E_{1}$;

(4) $0 \leq \omega^{\prime}(x)<+\infty$ for $x \in[a, b] \sim E_{1}$;

(5) $\omega(a)=0$ and $\omega(b)<\epsilon / 4$.

That such a function exists is well known; see Zahorski [30] or McGregor [16]. As $\omega$ is continuous in $[a, b]$, the corresponding interval function on $\sigma_{A}$, also denoted by $\omega$, belongs to $\bar{\pi}(A, \beta)$ by $(\bar{\pi} 6)$. Set $T=M+\omega, t=m-\omega$. Then $T \in \bar{\pi}(A, \beta)$ and $t \in \mathbb{\pi}(A, \beta)$.

Let $C$ be the set of points $x$ on which $\underline{D} M(x)>-\infty$ fails to hold. For $x \epsilon$ $E_{1} \sim C$

$$
\underline{D} T(x) \geq \underline{D} M(x)+D \omega(x)=+\infty,
$$

so that

$$
\mathfrak{D} T(x)=+\infty \geq f(x) \text {. }
$$

For $x \in A \sim\left[E_{1} \cup C\right], D_{M}(x)$ exists and is finite, so that $\mathscr{D} T(x)$ exists and is finite, and

$$
\mathscr{D} T(x)=\mathscr{D} M(x)+D \omega(x) \geq \underline{D} M(x) \geq f(x) .
$$

Similarly, $\mathfrak{D}_{t}(x)$ exists n.e. in $A$ and is finite in $A$, and $+\infty \neq \mathscr{D}(t, x) \leq f(x)$ n.e. in $A$. Furthermore,

$$
T(A)-t(A)=M(A)+\omega(A)-m(A)+\omega(A)<\epsilon .
$$

Thus, $T, t$ satisfy all the required conditions, and hence the proof is completed.

Corollary. Let $P_{1}=\left\{\bar{\pi}, \underline{\mathscr{I}}_{1}, \mathfrak{B}, \pi, \bar{I}\right\}$ be another such derivate system on $\sigma$ with $\mathfrak{D}_{1} M(x)=\mathscr{D}_{M}(x)$ n.e. in $A$ whenever one of $\mathscr{D}_{1} M(x), \mathscr{D}_{M}(x)$ exists n.e. in $A$. Then $P(A, \beta)=P_{1}(A, \beta)$ and two integrals of the same function are equal.

By Theorem 5, we see that

(I) we can use the $\mathfrak{D}$-derivatives instead of $\mathfrak{D}$-derivates in the definition of major functions and minor functions;

(II) the "almost everywhere" in $(\bar{M} 2)$ and $(\underline{m} 2)$ can be replaced by "nearly everywhere".

Statement (II) is well known for most of the particular integrals of Perron type while statement $(\mathrm{I})$ is due to McGregor [16] for the classical Perron integral. The proof here is essentially that of [16]. For a similar result for the $P^{n}$-integral, see Bullen [2]. We will use Theorem 5 and its corollary to prove the equivalence of the $S C P$-integral and the $M Z$-integral in later examples.

If the derivate system does not satisfy some extra conditions, one cannot get any reasonable integration by parts formula; but with some reasonably mild restrictions, which are unfortunately hard to check in particular cases, we have 
Theorem 6. Let $f \in P([a, b[, \beta)$ and $U$ be a bounded nonnegative point function on $[a, b], U^{\prime}(x)$ exist and be nonnegative a.e. in $[a, b]$, and such that the following inequalities make sense and are valid for eacb $M \in \bar{M}_{f}([a, b[, \beta), m \epsilon$ $\underline{M}_{f}([a, b[, \beta)$.

$$
\begin{aligned}
& \underline{\mathscr{D}}(M U)(x) \geq M(x) U^{\prime}(x)+U(x) \underline{\mathscr{D}} M(x) \text { a.e. in }[a, b], \\
& \overline{\mathfrak{D}}(m U)(x) \leq m(x) U^{\prime}(x)+U(x) \overline{\mathfrak{D}} m(x) \text { a.e. in }[a, b], \\
& \underline{\mathfrak{D}}(M U)(x)>-\infty \text { n.e. in }[a, b], \\
& \overline{\mathscr{D}}(m U)(x)<+\infty \text { n.e. in }[a, b] .
\end{aligned}
$$

Then if $F$ is the primitive of $f, f U+F U^{\prime} \in P\left(\left[a, b[, \beta)\right.\right.$, and $\int_{[a, b}\left[\left(f U+F U^{\prime}\right)=\right.$ $F\left(\left[a, b[) \cdot U\left(\left[a, b[)\right.\right.\right.\right.$. If, in addition, $F U^{\prime} \in P([a, b[, \beta)$ then so is $f U$ and

$$
\int_{[a, b[} f U=F\left(\left[a, b[) U\left(\left[a, b[)-\int_{[a, b[} F U^{\prime} .\right.\right.\right.\right.
$$

Proof. Under the hypotheses, one can easily see that if $M \in \overline{\mathbb{M}}_{f}$, then $M U \epsilon$

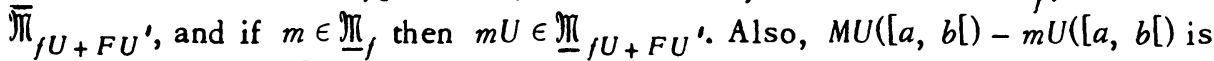
small when $M([a, b[)-m([a, b[)$ is small. Hence the required result follows easily.

Now, we are in a position to give another proof of Theorem 3 for the derivate

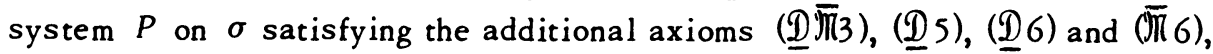
and also that a function $F$ satisfies $\bar{I}$ whenever $\underline{D} F(x)>-\infty$ except perhaps for a countable set of points.

Lemma 5. If a function is L-integrable, it is P-integrable and two integrals are equal.

Proof. By $(\bar{M} 6)$ and property (G), if a function is classical Perron integrable (see $\$ 6$ ), it is $P$-integrable and the two integrals are equal. It is well known that if a function is Lebesgue integrable, it is classical Perron integrable and the two integrals are equal. The conclusion then follows.

Lemma 6. Let $f(x) \geq 0$ almost everywhere in $[a, b]$. Then $f$ is the L-integrable on $[a, b]$ if and only if $f$ is P-integrable on $[a, b[$ with a base $B \epsilon$ $\mathfrak{B}([a, b[)$.

Proof. One direction is by Lemma 5. To prove the other direction, let $f$ be $P$-integrable on $[a, b[$ with a base $B$. Since $|f|=f$ almost everywhere in $[a, b]$, it follows that $|f|$ is also $P$-integrable on $[a, b]$ with base $B$. Clearly, the zero function $0 \in \mathbb{M}_{|f|}\left(\left[a, b[, B)\right.\right.$. Let $M \in \bar{\pi}_{|f|}([a, b[, B)$. Then by Lemma $1, M(=M-0)$ is monotone increasing in $B$. Define $M_{1}(x)=M(x)$ for $x \in B, M_{1}(x)=$ $\left.\sup _{t \in B \cap[a, x}\right]^{M(t)}$ for $x \in[a, b] \sim B$. Then $M_{1}$ is monotone increasing in $[a, b]$, so that $M_{1}^{\prime}(x)$ is $L$-integrable on $[a, b]$ and hence so is $\mathfrak{D}_{M}$ since $M_{1}^{\prime}(x)=\mathscr{D}_{M}(x)$ 
almost everywhere in $[a, b]$ by $(\underline{D} \bar{\pi} 1)$ and (D) 6$)$. As $f$ is measurable by Corollary 1 to Theorem 4, it follows that $f$ is $L$-integrable on $[a, b]$ since $|f(x)| \leq \mathscr{D}_{M}(x)$ almost everywhere in $[a, b]$. The proof is hence completed.

Corollary. Let $f_{1}$ be P-integrable on $\left[a, b\left[\right.\right.$ with base $B$, and $f_{2}$ be L-integrable on $[a, b]$ and $f_{1} \geq f_{2}$ almost everywhere in $[a, b]$. Then $f_{1}$ is also L-integrable on $[a, b]$.

Theorem $3^{\prime}$. Let $g, b, f_{n}(n=1,2,3, \ldots)$ be P-integrable on $[a, b]$ with a base $B$, and $g(x) \leq f_{n}(x) \leq b(x)$ almost everywhere in $[a, b]$ for eacb $n$, and $\lim _{n} f_{n}(x)=f(x)$ almost everywhere in $[a, b]$. Then $f$ is P-integrable on $[a, b]$ with base $B$ and $\int f=\lim _{n} \int f_{n}$.

Proof. Since $0 \leq f_{n}(x)-g(x) \leq b(x)-g(x)$ almost everywhere in $[a, b]$, both $f_{n}-g$ and $b-g$ are $L$-integrable on $[a, b]$ by Lemma 6 . By Lebesgue dominated theorem, we have

Hence, by Lemma 5 ,

$$
(L)-\int\left(f_{n}-g\right)=(L)-\int(f-g) \text {. }
$$

$$
\lim _{n}(P)-\int\left(f_{n}-g\right)=(P)-\int(f-g) \text {. }
$$

Now, $g$ is $P$-integrable, so that $f=(f-g)+g$ is also $P$-integrable and $\lim _{n}(P)-\int f_{n}=(P)-\int f$, completing the proof.

We close this section by remarking that Kubota's abstract integral of Perron type [13] is a particular case of the integral in this section. In fact, taking $B(A)=$ $\left\{\sigma_{A}\right\}, \pi=\{\varnothing\}$ and the inequality property $\bar{I}$ to mean $\underline{D} F(x)>-\infty$, one gets Kubota's setting and his integral if axiom (D) 4 ) is replaced by the equivalent axiom:

$\left(\underline{D} 4^{\prime}\right) \underline{D}\left(v_{1}+v_{2}\right)(x) \geq D v_{1}(x)+\underline{D} v_{2}(x)$ whenever the ordinary derivative $D v_{1}(x)$ exists.

Axioms (D4) and $\left(\mathfrak{D} 4^{\prime}\right)$ are equivalent in the sense that one follows from the

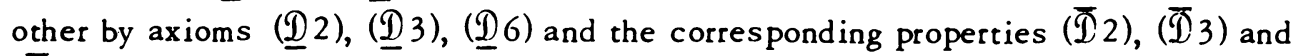

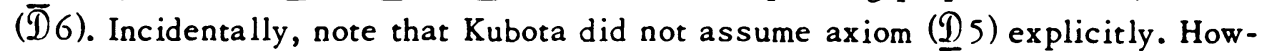
ever, in proving a result corresponding to our Lemma 6 , he did use implicitly (see the second last sentence in his proof of Theorem 3.8 [13]) our Corollary 1 to Theorem 4 , and axiom $(\mathfrak{D} 5)$ is essential in the proof of this corollary.

6. Examples. Some integrals defined in certain abstract spaces have been illustrated in $\$ 4$. In this section, we give some concrete integrals defined in the real line as examples. As in $\$ 5, X$ is to be the real line, $\sigma$ the family of all bounded half-open intervals like $[a, b[$, and $\pi$ the family of all subsets of $X$ with zero Lebesgue measure. Also, the interval functions are all to be additive, and hence are not to be distinguished from the point functions as we have mentioned in $\$ 5$. 
6.1. The scale of $C P$-integrals. For each derivate system to be defined in this subsection, the base mapping $\mathfrak{B}$ will be the same and is to be defined as $\mathscr{B}(A)=$ $\left\{\sigma_{A}\right\}$ for each. $A \in \sigma$. Legitimate mappings and derivate operators will be defined by induction. Once a lower derivate operator $\mathscr{D}$ is defined, a function $F$ will be said to satisfy the property $\bar{I}$ if and only if $\underline{D} F(x)>-\infty$ except for a countable set of points. Note that for all lower derivate operators used below, the property $\bar{I}$ so defined is an inequality property.

For each $A=\left[a, b\left[\right.\right.$, let $\pi^{0}(A)=\{M \mid M$ is continuous in $[a, b]\}$. Denoting $C_{0} D M(x)$ the ordinary lower derivate of $M$ at $x$, we let $P_{0}=\left(\pi^{0}, C_{0} D, \mathscr{B}, \pi, \bar{l}\right)$. Then $P_{0}$ is a derivate system on $\sigma$. The $P_{0}$-integral is just the ordinary Perron integral [22], [17]. Suppose that for each $n \geq 1$, the derivate system $P_{n-1}=$ $\left(\bar{\pi}^{n-1}, C_{n-1} D, B, \pi, \bar{I}\right)$ has been defined. For each $M \in P_{n-1}([a, b[)$ and for each $x \in[a, b]$ and $b \neq 0$ with $x+b \in[a, b]$, let

$$
C_{n}(M ; x, x+b)=\frac{n}{b^{n}}\left(P_{n-1}\right)-\int_{x}^{x+b}(x+b-t)^{n-1} M(t) d t .
$$

Then $M$ is said to be $C_{n}$-continuous at $x$ if $\lim _{b \rightarrow 0} C_{n}(M ; x, x+b)=M(x)$. Let

$$
\bar{\pi}^{n}\left(\left[a, b[)=\left\{M \mid M \text { is } C_{n} \text {-continuous in }[a, b]\right\},\right.\right.
$$

and for each $M$ define

$$
C_{-n} D M(x)=\liminf _{b \rightarrow 0} \frac{n+1}{b}\left\{C_{n}(M ; x, x+b)-M(x)\right\} .
$$

Then it can be shown that $P_{n}=\left(\vec{\pi} \cdot n, C_{n} D, \Re, \pi, \bar{I}\right)$ is a derivate system on $\sigma$. The $P_{n}$-integral is in fact equivalent $\frac{n}{(s e e}$ Bosanquet [1]) to the $C_{n} P$-integral of Burkill in [3], [4].

That $P_{n}$ is in fact a derivate system on $\sigma$ follows easily from Theorem 2.2 in [4]. For the theorem, there is a defect in Burkill's original proof (see [4, line 9 , p. 546]). This defect was noted recently and independently by Verblunsky in [28]. For a correct proof, we refer to [29] or [23].

6.2. A scale of approximately mean-continuous integrals. Ellis, starting from the general Denjoy integral has defined a scale of mean-continuous integrals [8], which is more general than the scale of $C P$-integrals ([3], [4], or subsection 6.1). However, Ellis' $M_{n}$-continuity and Burkill's $C_{n}$-continuity are equivalent for functions defined on intervals (see [24]). This leads us to consider a scale of approximately mean-continuous integrals.

First, we recall some concepts to be used. Generalizing the concept of AC functions, we say that a function $F$ is $\underline{\mathrm{AC}}$ (cf. [20]) on a set $E$ if for each $\epsilon>0$ there exists a $\delta>0$ such that $\Sigma_{r}\left\{F\left(b_{r}\right)-F\left(a_{r}\right)\right\}>-\epsilon$ for all finite sets $\left\{\left[a_{r}, b_{r}\right]\right\}$ of nonoverlapping intervals with endpoints in $E$ and with $\Sigma_{r}\left(b_{r}-a_{r}\right) \leq \delta$. A function is said to be ( $\underline{A C G}$ ) on a set $E$ if $E$ is a union of countably many closed sets on each of which the function is $\underline{A C}$. Note that $\underline{(A C G)}$ is an inequality property as defined in $\$ 1$. 
For $A \in \sigma$, letting $M$ be a function defined on $A$, we denote

$$
\underline{A D} M(x)=\text { app } \liminf _{b \rightarrow 0, x+b \in \bar{A}} \frac{M(x+b)-M(x)}{b},
$$

the lower approximate derivate of $M$ at $x$.

Now, replacing $C_{0} D$ by $\underline{A D}$, and $\bar{I}$ by $(\underline{A C G})$ in the derivate system $P_{0}$ in subsection 6.1 , we obtain a new derivate system, say $A M_{0} P=\left(\bar{\pi}^{0}, A D, \mathfrak{B}, \mathcal{r}\right.$, $($ ACG $)$. The $A M_{0} P$-integral is in fact equivalent to the general Denjoy integral ([20]).

Suppose that for each $n \geq 1$, the derivate system $A M_{n-1} P=\overline{(A)}^{n-1}, \underline{A D}, \mathfrak{B}$, $\pi,(\underline{A C G}))$ has been defined, where $\overline{A^{0}}{ }^{0}=\bar{M}^{0}$. We say that a function $F$ is $A M_{n}$ continuous at $x$ if $F$ is $A M_{n-1} P$-integrable on an interval containing $x$ and

Define

$$
\underset{b \rightarrow 0}{\operatorname{app}} \lim \frac{n}{b^{n}}\left(A M_{n-1} P\right)-\int_{x}^{x+b}(x+b-t)^{n-1} F(t) d t=F(x) \text {. }
$$

$$
\bar{A}^{n}(A)=\left\{M \mid M \text { is } A M_{n} \text {-continuous in } \bar{A}\right\}
$$

for each $A \in \sigma$, and let

$$
\left.A M_{n} P=\left(\bar{A}^{\pi}\right)^{n}, \underline{A D}, \mathfrak{B}, \Re,(\underline{A C G})\right) .
$$

Thus, inductively, we have defined $A M_{n} P$ for $n=0,1,2,3, \ldots$. It can be proved in a very similar way to the case of $P_{n}$ in subsection 6.1 that each $A M_{n} P$ is in fact a derivate system on $\sigma$. We call the $A M_{n} P$-integral, $n=0,1,2,3, \cdots$, a scale of approximately mean-continuous integrals.

The general property of $A M_{n} P$-integral follows from our general theory. In addition, one can prove that the scale is more general than both scales by Burkill and by Ellis; the consistency of the scale and also an integration by parts formula can be obtained similar to those of Burkill's CP-scale and Ellis' GM-scale.

Remarks. (1) We might define in a similar way another scale of integrals by taking the ordinary continuity in the definition of $\overline{A M}^{0}=\bar{\pi}^{0}$ to be the approximate continuity. However, doing this we are unable to obta in the consistency of the new scale.

(2) For $n=1,2,3, \cdots$, let $\bar{\pi}^{n}(A)=\left\{M \mid M\right.$ is $M_{n}$-continuous in $A$ (in the sense of Ellis [8])\}, and let

$$
M_{n} P=(\bar{\pi} n, \underline{A D}, \mathcal{B}, \Re,(\underline{A C G})) .
$$

Then $M_{n} P$ is a derivate system and the $M_{n} P$-integral can be proved to be equivalent to Ellis' $G M_{n}$-integral, which was defined originally by a descriptive method of Denjoy.

6.3. A scale of symmetric CP-integrals. Burkill has defined a SCP-integral in [5], which is more suitable for application to trigonometric series. Although, the $S C P$-integral has been investigated by many people, no "symmetric" scale corresponding to the $C P$-scale has appeared in the literature. We show briefly how 
to obtain such a symmetric scale from our general theory. We study this scale in greater detail in another paper.

For each integer $n \geq 1$, we start from the $C_{n-1} P$-integral. Generalizing the concept of $C_{n}$-continuity, we say that a function $M$ is $S C_{n}$-continuous at $x$ if

$$
\lim _{b \rightarrow 0}\left\{C_{n}(M ; x, x+b)-C_{n}(M ; x, x-b)\right\}=0 .
$$

We also define

$$
\underline{S C_{n} D} M(x)=\liminf _{b \rightarrow 0_{+}} \frac{n+1}{2 b}\left\{C_{n}(M ; x, x+b)-C_{n}(M ; x, x-b)\right\}
$$

and we say that $M$ satisfies the property $\bar{I}_{n}$ if $S C_{n} D M(x)>-\infty$ except perhaps for points of a scattered set. For the definition and properties of scattered sets, we refer to [14] and [12]. For an interval $[a, b[$, we let $\mathfrak{B}([a, b[)=$ the family of all bases in $[a, b]$, i.e. $B \in \mathcal{B}([a, b[)$ if and only if $B \subset[a, b]$ with measure $b-a$ and $a, b \in B$ (cf. $\S 5)$. Then, for each $B \in \mathcal{B}([a, b[)$, let

$\overline{s \pi n}^{n}\left(\left[a, b[, B)=\left\{M \mid M\right.\right.\right.$ is $C_{n}$-continuous in $B$ and $S C_{n}$-continuous in $] a, b[\}$.

Define

$$
S C_{n} P=\left(\overline{S \pi^{n}}, \underline{S C_{n} D}, \Re, \Re, \bar{I}_{n}\right) .
$$

Then $S C_{n} P$ is a derivate system on $\sigma$, which furthermore satisfies the additional axioms $\left(\underline{D}_{5}\right),(\underline{D} 6),\left(\bar{\pi}_{6}\right),\left(\bar{I}_{4}\right)$ in $\S 5$, and also $\left(\bar{\pi}_{5}^{\prime}\right)$ in $\S 3$. For $n=1,2,3, \cdots$, the $S C_{n} P$-integrals are called a scale of symmetric $C P$-integrals, which is more general than Burkill's scale of CP-integrals.

Similar to the $C P$-scale, the $S C P$-scale also has the consistency theorem, i.e. if $f$ is $S C_{n} P$-integrable, it is also $S C_{n+1} P$-integrable and two integrals are equal. Although the $S C_{n} P$-integral is not as general as James' $P^{n+1}$-integral [11], [12], the $S C_{n} P$-integral is additive as an interval function (by property (C) in $\$ 2$ ), while $P^{n+1}$-integral fails to have this property (cf. [25] and [7]).

We note that for this derivate system $S C_{1} P$, if we replace the inequality prop-

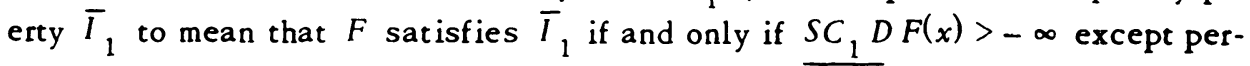
haps for points of a countable set, then we get a new derivate system, denoted by $S C P$. Then the SCP-integral is just that defined by Burkill in [5]. However, SCP. and $S C_{1} P$-integrals are equivalent due to the remark at the end of [12].

6.4. The MZ-integral and the SCP-integral. We obtain a MZ-integral and a $S C P_{R}$-integral and prove that both are equivalent to the $S C P$-integral of Burkill [5]. We take $B, S^{1}$ and $S C_{1} D$ as those in subsection 6.3 , and consider a new legitimate mapping $\overline{S \pi_{R}} \overline{\text { defined by }}$ 


$$
\begin{aligned}
& \overline{S \mathbb{M}_{R}}(A, B)=\left\{M \mid M \in \overline{S M}^{1} \text { and, for each } b>0 \text { with } x \pm b \in A,\right. \\
& \lim _{\epsilon \rightarrow 0+} \int_{\epsilon}^{b} \frac{M(x+u)-M(x-u)}{2 u} d u \text { exists (finite or infinite) } \\
& \text { except for points } x \text { in a countable set\}. }
\end{aligned}
$$

For each $A=[a, b[$ and $M \in \overline{S \pi R}(A, B)$, define

$$
\begin{aligned}
& \underline{B}_{s} M(x)=\liminf _{b \rightarrow 0+} \frac{1}{b} \int_{0}^{b} \frac{M(x+u)-M(x-u)}{2 u} d u \text { for } x \in[a, b[, \\
& \underline{B}_{s} M(a)=\underline{B} M(a)=\liminf _{b \rightarrow 0+} \frac{1}{b} \int_{0}^{b} \frac{M(a+u)-M(a)}{u} d u, \\
& \underline{B}_{s} M(b)=\underline{B} M(a)=\liminf _{b \rightarrow 0+} \frac{1}{b} \int_{0}^{b} \frac{M(b)-M(b-u)}{u} d u .
\end{aligned}
$$

$B_{s} M(x)$ is called the symmetric lower Borel derivate of $M$ at $x$. Let

$$
\begin{aligned}
S C P_{R} & =\left(\overline{S M R}, S C_{1} D, \Re, \Re, \bar{I}\right) \text { and } \\
M Z & \left.=\overline{(S) \pi R}, \overline{B_{s},} \mathfrak{B}, \Re, \bar{I}\right),
\end{aligned}
$$

where $\bar{I}$ is defined to be that $F$ satisfies $\bar{I}$ if and only if $S C_{1} D F(x)>-\infty$ (resp. $B_{s} F(x)>-\infty$ ) except perhaps for points of a countable set. Then $S C P_{R}$

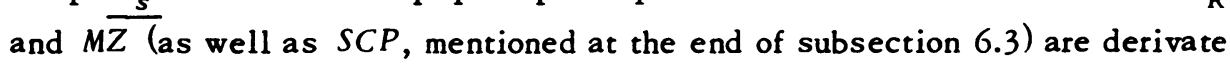
systems on $\sigma$, and satisfy all the extra axioms in $\S 5$, except that $\overline{s \pi_{R}}([a, b[, B)$ may not contain all the functions continuous in $[a, b]$. However, one can prove that the function $\omega$ used in the proof of Theorem 5 belongs to $\overline{S \pi_{R}}([a, b[, B)$. Thus, all the results in $\$ 5$ are applicable to the $S C P-, S C P_{R^{-}}$and $M Z$-integrals. We note that the $M Z$-integral is just that of Marcinkiewicz and Zygmund [15] except that the latter was defined by using Lebesgue integral instead of Perron integral.

Now, we establish two lemmas, which will be used to prove the equivalences of $M Z$-, $S C P_{R^{-}}$and $S C P$-integrals.

Lemma 7. Let $M \in \bar{S} \overline{M_{R}}\left(\left[a, b[, B)\right.\right.$. Then $B_{s} M(x)$ exists if and only if $S C_{1} D M(x)$ exists, and the two are equal if they exist.

Proof. Let $M_{1}$ be the Perron primitive of $M$. Then it is easy to check that $S C_{1} D M(x)=D_{2} M_{1}(x)$ and $\overline{S C_{1} D M}(x)=\bar{D}_{2} M_{1}(x)$, where

$$
\underline{D}_{2} M_{1}(x)=\lim \inf \frac{M_{1}(x+b)+M_{1}(x-b)-2 M(x)}{2 b}
$$


and $\bar{D}_{2} M_{1}$ is defined similarly. The conclusion then follows from Lemma 28 in [15].

Lemma 8. Let $M \in \bar{S}^{1}\left(\left[a, b[, B)\right.\right.$ and $S C_{1} D M(x)$ exist except perbaps for points of a countable set. Then $M \in \overline{S \mathbb{M} R_{R}}([a, b[, B)$.

Proof. Let $S C_{1} D M\left(x_{0}\right)$ exist and let

$$
\psi(u)=\int_{0}^{t}\left\{M\left(x_{0}+u\right)-M\left(x_{0}-u\right)\right\} d u .
$$

For $0<k<b$,

$$
\begin{aligned}
\int_{k}^{b} \frac{M\left(x_{0}+u\right)-M\left(x_{0}-u\right)}{2 u} d u & =\int_{k}^{b} \frac{\psi^{\prime}(u)}{2 u} d u \\
& =\frac{1}{2}\left\{\frac{\psi(b)}{b}-\frac{\psi(k)}{k}+\int_{k}^{b} \frac{\psi(u)}{u^{2}} d u\right\}
\end{aligned}
$$

by integration by parts. By the $S C_{1}$-continuity of $M, \psi(k) / k \rightarrow 0$ as $k \rightarrow 0+$. For $S C_{1} D M\left(x_{0}\right)$ finite, $\psi(u) / u^{2}$ is bounded for small $u$; for $S C_{1} D\left(x_{0}\right)= \pm \infty$, $\psi(u) / u^{2}$ is of constant sign for small $u$. In all cases, one sees that $\lim _{k \rightarrow 0+} \int_{k}^{b} \psi(u) / u^{2} d u$ exists, so that $\lim _{k \rightarrow 0+} \int_{k}^{b}\left(M\left(x_{0}+u\right)-M\left(x_{0}-u\right)\right) / 2 u d u$ exists (finite or infinite), completing the proof.

Theorem 7. The SCP-, $S C P_{R^{-}}$and MZ-integrals are all equivalent.

Proof. By Lemma 7, one sees that the corollary to Theorem 5 applies to the derivate systems $S C P_{R}(=P)$ and $M Z\left(=P_{1}\right)$, so that the $S C P_{R^{-}}$and the $M Z$-integral are equivalent. To see that they are also equivalent to the $\frac{R}{S C} \bar{P}$-integral, note that by the property $(G)$ in $\$ 2$, the $S C P$-integral is more general than the $S C P_{R}$-integral. It remains to show that the $M Z$-integral is more general than the $S C P$-integral. To do this, let $f$ be a $S C P$-integrable function. Applying Theorem 5 to the derivate system $S C P$, one obtains for $f$ an appropriate $S C P$-major function $T$ and an appropriate $S C P$-minor function $t$. Then by Lemma 8 and Lemma 7 , one sees that $T, t$ are respectively relevant $M Z$-major and minor functions for $f$, so that it is $M Z$-integrable, completing the proof.

We remark that the integration by parts formula for $S C P$-integral stated by Burkill in [5] remains unproved. Hence his proof of Theorem 5.2 in [5] (the SCP. integral solves the coefficient problem of the convergent trigonometric series! breaks down. However, this theorem remains true by our Theorem 7 since it has been proved in [15] that the $M Z$-integral solves the coefficient problem. We remark that the proof in [15], without using integration by parts but using formal multiplication of series (cf. also James [10]), applies to the SCP-integral, too.

Acknowledgment. Special thanks are due to the referee for his valuable suggestions and careful reading. 


\section{REFERENCES}

1. L. S. Bosanquet, A property of Cesàro-Perron integrals, Proc. Edinburgh Math. Soc. (2) 6 (1940), 160-165. MR 2, 131.

2. P. S. Bullen, The $P^{n}$-integral, J. Austral. Math. Soc. 14 (1972), 219-236.

3. J. C. Burkill, The Cesàro-Perron integral, Proc. London Math. Soc. (2) 34 (1932), 314-322.

4. - The Cesàro-Perron scale of integration, Proc. London Math. Soc. (2) 39 (1935), 541-552.

5. - Integrals and trigonometric series, Proc. London Math. Soc. (3) 1 (1951), 46-57. MR 13, 126.

6. G. E. Cross, The relation between two definite integrals, Proc. Amer. Math. Soc. 11 (1960), 578-579. MR $22 \# 8094$.

7. - On the generality of the AP-integral, Canad. J. Math. 23 (1971), 557-561. MR $44 \# 378$.

8. H. W. Ellis, Mean-continuous integrals, Canad. J. Math. 1 (1949), 113-124.

MR 10,520 .

9. R. Henstock, Generalized integrals of vector-valued functions, Proc. London Math. Soc. (3) 19 (1969), 509-536. MR $40 \# 4420$.

10. R. D. James, A generalized integral. II, Canad. J. Math. 2 (1950), 297-306. MR 12, 94.

11. - Generalized nth primitives, Trans. Amer. Math. Soc. 76 (1954), 149-176. MR 15, 611 .

12. - Summable trigonometric series, Pacific J. Math. 6 (1956), 99-110.

MR 17, 1198.

13. Y. Kubota, A generalized derivative and integrals of Perron type, Proc. Japan Acad. 41 (1965), 443-448. MR $32 \# 1321$.

14. K. Kuratowski, Topologie. Vol. I, PWN, Warsaw, 1958; English transl., Academic Press, New York; PWN, Warsaw, 1966. MR 19, 873; MR 36 \#840.

15. J. Marcinkiewicz and $A$. Zygmund, $O$ n the differentiability of functions and summability of trigonometric series, Fund. Math. 26 (1936), 1-43.

16. J. C. McGregor, An integral of Perron type, Thesis, University of British Columbia, 1951 (unpublished).

17. E. J. McShane, Integration, Princeton Univ. Press, Princeton, N. J., 1944. MR 6, 43.

18. - A Riemann integral that includes Lebesgue-Stieltjes, Bochner, and stochastic integrals, Mem. Amer. Math. Soc. No. 88 (1969). MR 42 \#436.

19. W. F. Pfeffer, An integral in topological spaces. I, J. Math. Mech. 18 (1969), 953972. MR $39 \# 2938$.

20. J. Ridder, Über den Perronschen Integralbegriff und seine Beziehung zu den R-, L- und D-integration, Math. Z. 34 (1931), 234-269.

21. P. Romanovski, Intégrale de Denjoy dans des espaces abstraits, Mat. Sb. 9 (51) (1941), 67-120. MR 2, 354.

22. S. Saks, Theory of the iniegral, 2nd rev. ed., Monografie Mat., vol. 7, PWN, Warsaw, 1937.

23. W. L. C. Sargent, On sufficient conditions for a function integrable in the CesàroPerron sense to be monotonic, Quart. J. Math. Oxford Ser. 12 (1941), 148-153. MR 3, 228. 
24. W. L.C. Sargent, Some properties of $C_{\lambda}$-continuous functions, J. London Math. Soc. 26 (1951), 116-121. MR 12, 810.

25. V. A. Skvorcov, Concerning definitions of $P^{2}$ and SCP-integrals, Vestnik Moskov. Univ. Ser. I Mat. Meh. 21 (1966), no. 6, 12-19. MR 34 \#7765.

26. D. W. Solomon, Denjoy integration in abstract spaces, Mem. Amer. Math. Soc. No. 85 (1969). MR 39 \#404.

27. S. J. Taylor, An integral of Perron's type with help of trigonometric series, Quart. J. Math. Oxford Ser. (2) 6 (1955), 255-274. MR 19, 255.

28. S. Verblunsky, On a descriptive definition of Cesàro-Perron integrals, J. London Math. Soc. (2) 3 (1971), 326-333. MR 44 \#4161.

29. - On the Peano derivatives, Proc. London Math. Soc. (3) 22 (1971), 313324. MR 44 \#2896.

30. Z. Zahorski, Über die Menge der Punkte in welchen die Ableitung unendlich ist, Tôhoku Math. J. 48 (1941), 321-330. MR 10, 359.

DEPARTMENT OF MATHEMATICS, UNIVERSITY OF BRITISH COLUMBIA, VANCOUVER 8 , BRITISH COLUMBIA, CANADA

DEPARTMENT OF MATHEMATICS, UNIVERSITY OF WISCONSIN, MILWAUKEE, WISCONSIN 53201 